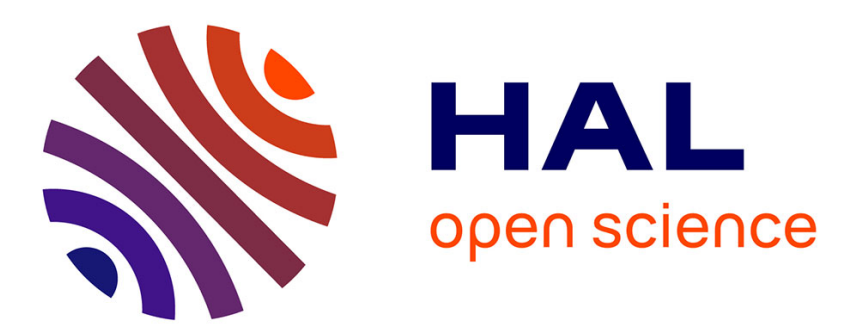

\title{
Statistical and temporal irradiance fluctuations modeling for a ground-to-geostationary satellite optical link
}

\author{
A.R. Camboulives, M.T. Velluet, S. Poulenard, L. Saint-Antonin, V. Michau
}

\section{To cite this version:}

A.R. Camboulives, M.T. Velluet, S. Poulenard, L. Saint-Antonin, V. Michau. Statistical and temporal irradiance fluctuations modeling for a ground-to-geostationary satellite optical link. Applied optics, 2018, 57 (4), pp.709-721. 10.1364/AO.57.000709 . hal-01717282

\section{HAL Id: hal-01717282 \\ https://hal.science/hal-01717282}

Submitted on 26 Feb 2018

HAL is a multi-disciplinary open access archive for the deposit and dissemination of scientific research documents, whether they are published or not. The documents may come from teaching and research institutions in France or abroad, or from public or private research centers.
L'archive ouverte pluridisciplinaire HAL, est destinée au dépôt et à la diffusion de documents scientifiques de niveau recherche, publiés ou non, émanant des établissements d'enseignement et de recherche français ou étrangers, des laboratoires publics ou privés. 


\title{
Statistical and temporal irradiance fluctuations modeling for a ground-to-geostationary satellite optical link
}

\author{
A.-R. Camboulives ${ }^{1,2, *}$, M.-T. Velluet ${ }^{2}$, S. Poulenard ${ }^{3}$, L. Saint-Antonin ${ }^{1,3}$, and V. Michau ${ }^{2}$ \\ ${ }^{1}$ IRT Saint-Exupery, 118 Route de Narbonne, Toulouse, France \\ ${ }^{2}$ ONERA-DOTA, 29 Avenue de la Division Leclerc, Châtillon, France \\ ${ }^{3}$ Airbus Defence and Space, 31 Rue des Cosmonautes, Toulouse, France \\ *Corresponding author: adrien-richard.camboulives@irt-saintexupery.com
}

Compiled December 14, 2017

\begin{abstract}
An optical communication link performance between the ground and a geostationary satellite can be impaired by scintillation, beam wandering and beam spreading due to its propagation through atmospheric turbulence. These effects on the link performance can be mitigated by tracking and error correction codes coupled with interleaving. Precise numerical tools capable of describing the irradiance fluctuations statistically and of creating irradiance time series are needed to characterize the benefits of these techniques and optimize them. The wave optics propagation method have proven their capability of modeling the effects of atmospheric turbulence on a beam but it is known to be computationally intensive. We present an analytical-numerical model that provides good results on the probability density functions of irradiance fluctuations as well as time series with an important saving of time and computational resources. (๑) 2017 Optical Society of America
\end{abstract}

OCIS codes: (010.1080) Active or adaptive optics; (010.1300) Atmospheric propagation;

\section{INTRODUCTION}

An optical link based on a multiplex of wavelengths around the $1.55 \mu \mathrm{m}$ spectral band is foreseen to be a valuable alternative to the conventional radio-frequencies feeder links for next generation broadband geostationary satellites, targeting a capacity of around 1Tbps. In addition to cloud obstruction, one of the major limitations to optical links is the presence of atmospheric turbulence during the first $20 \mathrm{~km}$ of the propagation. In this paper, we will focus on the ground-to-satellite link, or uplink.

During its propagation from a ground station to a geostationary satellite, the optical beam is deflected (beam wandering) and possibly distorted (beam spreading) by atmospheric turbulence. It induces strong fluctuations of the detected telecommunication signal, thus increasing the Bit Error Rate (BER). To correct these effects, the beam characteristics need to be modified at the emission (pre-compensation). The envisaged technique is adaptive optics (AO) in which a servo system modifies in real time the emitted wavefront in order to make it recover a plane waveform when reaching the satellite, using the reciprocity principle as envisioned by Fried and Yura [1]. To do so, the beam coming from the satellite will be used to estimate the perturbations that need to be applied to the emitted wavefront. However, there is a point-ahead angle between the downlink and the uplink in order for the uplink to intercept the moving satellite at the position where it will be when the pulse arrives. This means that the turbulence effects experienced by the downlink and the uplink are slightly different, leading to partial compensation only. Other effects also have an impact on the quality of the compensation, such as the optical ground station architecture through the beam size and aperture mismatch.

Because of the turbulence-induced irradiance fluctuations (the power detected by the satellite's terminal), the signal modulation detection and the link budget are disturbed. To evaluate the degradation, we need to estimate the probability density function and time series of the received signal. Wave optics simulations (in this article, we will use TURANDOT [2]) permit to obtain very precise results for the irradiance fluctuations statistics as well as time series. However, they require a lot of computational power due to the requirements on the phase screens sampling and sizing in the case of time series[3]. This leads to long duration simulations. Wave optics simulations are therefore not practical for realizing sensitivity studies with many parameters of the optical ground station and propagation channel or long time series. Hence, there is a need to obtain similar results to wave optics simulations while requiring less computational power.

There have been many studies aiming to give a thorough de- 
scription of the irradiance fluctuations on a ground-to-satellite propagation. These studies' objectives are usually to present analytical expressions for the scintillation index (the normalized irradiance variance), the irradiance probability density functions and even for the probability of fades $([4,5])$. These studies usually rely on the Rytov perturbation method (RPM) [6]. However, discrepancies appear for ground-to-space paths which are usually attributed to beam wander[7, 8]. Moreover, the temporal aspects of the irradiance fluctuations are usually described by the number of fades per second and the mean fade time. With these analytical approaches, it is not possible to create irradiance series and test performance of error. In order to obtain irradiance time series, we have relied on the existing work regarding the impact of the temporal effects of atmospheric turbulence on beam propagation ([9]).

The objective of this article is to present a model that performs time series of irradiance fluctuations as detected by the satellite, with and without the tracking system. In order to do so, we will base our work on an existing model from the literature ([10]) which gives statistical results of irradiance fluctuations in the case of a collimated beam propagating from the ground to a geostationary satellite. This model is called the Low-Order of Turbulence (LOT) model. We have compared the LOT model's results (cumulative density functions and temporal power spectral density functions) with wave optics simulations to discuss its validity. We have demonstrated that the LOT model is not as accurate as we need due to its lack of modeling the propagation of a beam through multiple phase screens. We propose a method to improve and expand it in order to take into account the tracking and obtain time series of irradiance fluctuations. The final model is called the WPLOT model (With Propagation Low Order of Turbulence model). All the models to which we will reference throughout the article are described in Appendix A.

The WPLOT model will depend on the parameters of the optical ground station and of the propagation channel, and will take into account the inherent errors within tracking. It should permit to optimize many parameters of an optical ground station architecture. The parameters required to evaluate the turbulence effect on the link performance are introduced in Section 2. In Section 3, the developed model is described. Results obtained with this model are compared with those obtained with wave optics simulations in Section 4. Finally, in Section5, we present results when tracking is taken into account.

\section{MODEL REQUIREMENTS}

Thanks to the optical link budget, an estimation of the general performance of the system can be assessed by estimating the received power detected by the satellite. On the other hand, knowledge of the temporal irradiance fluctuations will permit to study the viability of envisioned error correction codes coupled with interleaving.

\section{A. Optical link budget}

The optical link budget gives an estimation of the received power $P_{R}$ as a function of the emitted power $P_{E}$, taking into account all losses during the beam propagation: $P_{R}=$ $G_{R} L_{\text {TURB }} L_{\text {OTHERS }} G_{E} P_{E}$. $L_{\text {TURB }}$ are the losses induced by the irradiance fluctuations due to turbulence. $L_{O T H E R S}$ are those induced by other contributors such as transmission losses through the emitter and receiver, atmospheric absorption and scattering losses, cloud margin, fiber injection and the free- space losses. $G_{E}$ and $G_{R}$ are the gains respectively at the emission and reception taking into account the diameters of the telescopes at the emission and on the satellite (and the waist size at the emission).

Atmospheric turbulence is a random phenomenon and thus implies that detected irradiance fluctuations are random as well. A statistical approach is therefore considered, focusing on one quantity: the irradiance threshold $I_{T}$ defined by estimating the probability $P\left(I>I_{T}\right)=0.95$, where $I$ is the instantaneous detected irradiance. Finding $I_{T}$ provides the loss term $L_{T U R B}$ due to atmospheric turbulence at a $5 \%$-probability of the cumulative distribution function. The other losses $\left(L_{O T H E R S}, L_{F S}\right)$ in the optical link budget are set and static. The gains $G_{E}$ and $G_{R}$ can be easily determined[11].

In order to find the irradiance threshold $I_{T}$, we need to have access to the irradiance cumulative density function, and thus to the irradiance probability function.

\section{B. Temporal fluctuations}

The irradiance threshold is defined at a $5 \%$-probability of the cumulative distribution function. This means that the instantaneous irradiance will spend $5 \%$ of the time below the threshold. During these periods, interleaving (coupled with the error correction codes) will permit to mitigate the losses and significantly improve the BER. In order to optimize the codes, there is a need for realistic irradiance time series in order to estimate the probability density functions of the fading times, the number of fadings per second, etc. These statistics will be obtained with long time series of irradiance.

In conclusion, the model we aim to develop must be able to provide not only the cumulative density functions of the irradiance fluctuations but also be able to generate irradiance time series.

\section{MODEL DESCRIPTION}

We will consider a ground to satellite optical link that corresponds to a weak turbulence regime, for which the scintillation index $\left(\sigma_{I}^{2}=\frac{\left\langle I^{2}\right\rangle-\langle I\rangle^{2}}{\langle I\rangle^{2}}\right)$ is lower than 1. Baker [10] describes different regions of distinct behavior for Gaussian beam on-axis weak scintillation. In one region, named $D_{1}$, the Rytov perturbation method (RPM) fails to give an accurate description of the beam-wave scintillation[? ]. Baker proposes to bound this region using Eq. 18 with the metrics $N_{L}$ and $N_{\tau}$ defined respectively in Eq. 15 and 16 in Appendix B. These bounds imply that the majority of the turbulence is in the near-field of the beam while the satellite is in far-field. Due to the distance between the ground and the geostationary satellite $(36000 \mathrm{~km})$, the bound on $N_{L}$ is never predominant over the bound on $N_{\tau}$, which therefore bounds the minimum waist size $w_{0}$ at the emission as a function of the turbulence strength distribution along the path.

\section{A. Low-order turbulence solution}

Baker proposes to describe the irradiance fluctuations by estimating the deformations of a Gaussian beam propagating through the atmosphere with the first and second order Zernike polynomials $[12,13]$ : tip/tilt, defocus and astigmatism. This is the Low-Order of Turbulence (LOT) solution. The LOT's major results are recalled in Appendix B. This solution is equivalent to integrating the refractive index fluctuations present along the path into a single phase screen placed at the transmitter (Figure $1)$. 


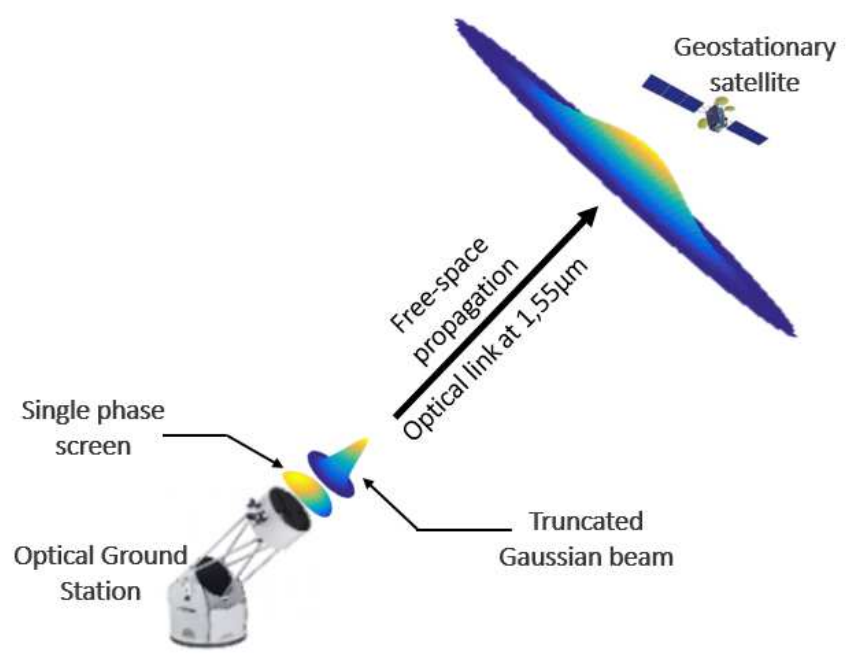

Fig. 1. Presentation of the LOT model. This model is equivalent to a single phase screen resulting from tilt, defocus and astigmatism placed in the emission plane of the beam.

\section{A.1. Effects of propagation and LOT solution}

The LOT solution assumes that the turbulence can be integrated in a single phase screen placed at the transmitter prior to propagation. This assumes that the effects of the propagation through multiple atmospheric layers are negligible inside the turbulence volume. In order to validate this assumption, we have compared in Figure 2 the detected irradiance results obtained with the LOT solution with results obtained after Fresnel propagation through phase screens evenly distributed along the propagation path. The phase screens are constructed as a linear combination of tip, tilt, defocus and astigmatism. The Zernike coefficients are obtained from the Noll variances in Eq. 23 and Eq.24 for the atmospheric parameters presented in Section 4. For this study, we will consider a Gaussian beam with a waist size $w_{0}=8 \mathrm{~cm}$. This leads to the metrics $N_{L}=3.6 \times 10^{-4}<1$ and $N_{\text {tau }}=9.4\left(N_{\text {tau }}^{2} \gg 1\right)$ being well within the $D_{1}$ region.

To illustrate the effects of the propagation through multiple phase screens, we will consider only a two layer atmosphere. The first layer is placed at the transmitter while the second layer is at a $10 \mathrm{~km}$ altitude. This will be called the $2 \mathrm{~L}-\mathrm{WO}$ simulation (for 2-Layer Wave Optics simulation). The objective of the $2 \mathrm{~L}-\mathrm{WO}$ simulation is to highlight the effects of the propagation through multiple atmospheric layers using the hypothesis of the LOT model that tip/tilt, defocus and astigmatism are sufficient to describe the beam deformations resulting from atmospheric turbulence in the $D_{1}$ region. Using only two layers is sufficient to make these effects appear.

In order to keep with the different used models in this article, Table 1 summarizes them with their specificities in Appendix A.

We observe, in Figure 2, a good correlation of the results even though some differences appear. Studying the effects of each optical aberration separately, we can see that for tip/tilt and astigmatism, there is a near-perfect fit whether one or more layers are used. However, differences appear for the defocus aberration, as can be seen in Figure 3.

An irradiance threshold appears for the LOT model compared to the $2 \mathrm{~L}-\mathrm{WO}$ simulation. This is because the focusing effects are limited by diffraction when using only one phase screen. The volume effects from propagating through multiple

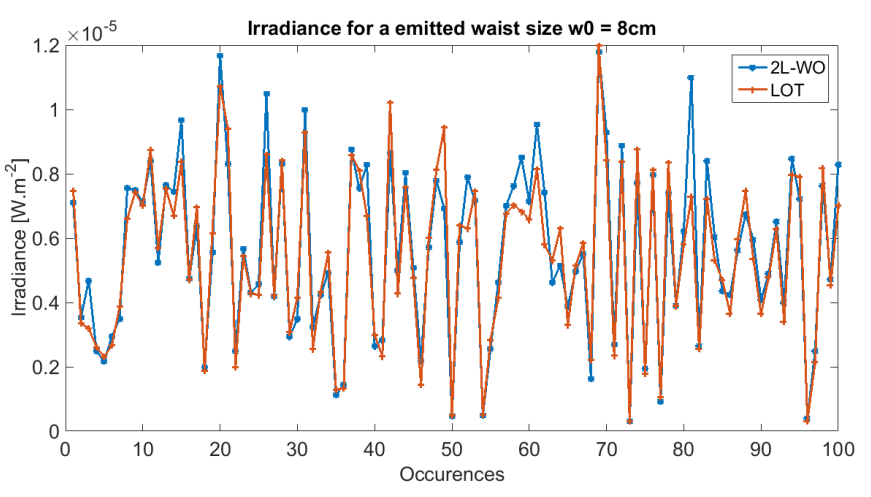

Fig. 2. Random draws of irradiance statistics between LOT model (1 layer) and the 2L-WO model, for an emitted waist of $8 \mathrm{~cm}$. In this case, $N_{L}=3.6 \times 10^{-4}$ and $N_{\text {tau }}=9.4$, well within the $D_{1}$ region.

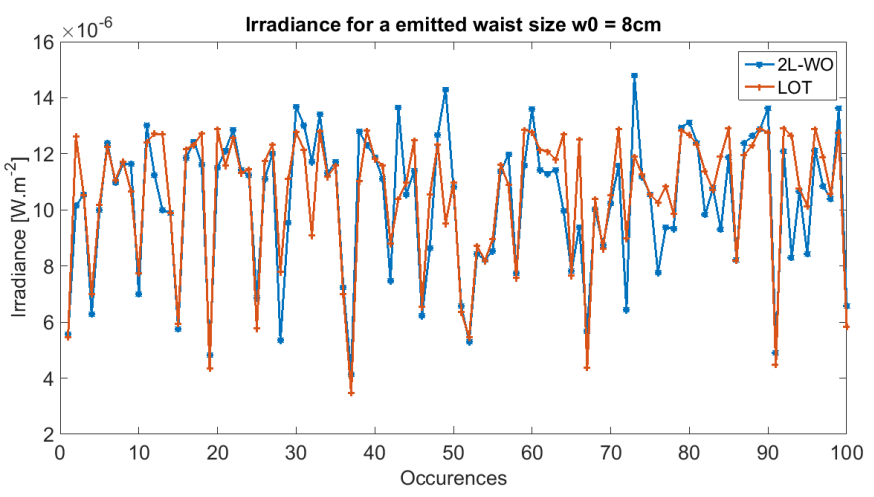

Fig. 3. Random draws of irradiance statistics between LOT solution and 2L-WO model when considering only defocus, for an emitted waist of $8 \mathrm{~cm}$. In this case, $N_{L}=3.6 \times 10^{-4}$ and $N_{\text {tau }}=9.4$, well within the $D_{1}$ region.

phase screens lead to a more focused beam than when using only one phase screen.

Figure 4 compares the Probability Density Functions (PDF) of irradiance for cases where only defocus is applied at 1,2 or 10 layers. It shows that using only one layer greatly reduces the dynamic of irradiance fluctuations: the PDF for only one layer is narrower than for multiple layers. However, the differences between 2 or 10 layers are not significant. This means that at least 2 atmospheric layers should be used.

In order to take into account the propagation through multiple phase screens, we propose to use the ABCD matrix propagation method [4], where the ABCD matrix is obtained from

$$
\left(\begin{array}{ll}
A & B \\
C & D
\end{array}\right)=\left(\begin{array}{cc}
1 & L_{\text {prop }} \\
0 & 1
\end{array}\right) \prod_{i}^{N}\left(\begin{array}{cc}
1 & 0 \\
\Delta \kappa_{i} & 1
\end{array}\right)\left(\begin{array}{cc}
1 & \Delta z_{i} \\
0 & 1
\end{array}\right),
$$

where $\Delta \kappa_{i}$ is the curvature (in meters ${ }^{-1}$ ) resulting from defocus at the $i^{\text {th }}$ layer, $\Delta z_{i}$ is the distance between two successive phase screens $i$ and $i+1$ and $N$ is the total number of considered layers. $L_{\text {prop }}$ is the remaining distance to the satellite: $L_{\text {prop }}=L-\sum_{i}^{N} \Delta z_{i}$, where $L$ is the distance between the ground and the satellite $(L=36000 \mathrm{~km})$. Usually $\sum_{i}^{N} \Delta z_{i}$ is of the order of $20 \mathrm{~km}$.

Considering the following initial complex radius of curva- 


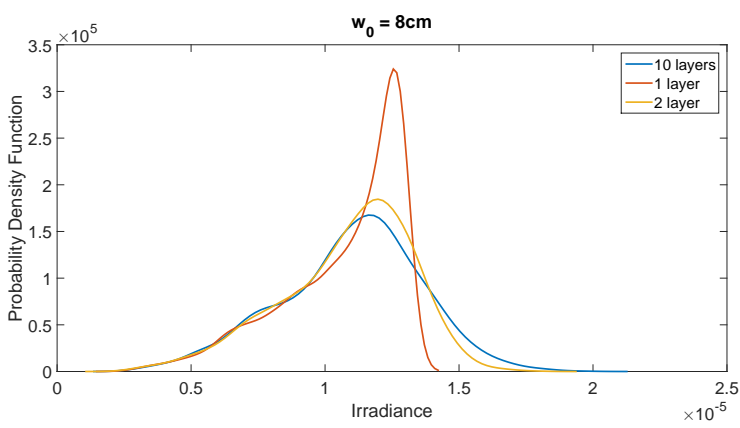

Fig. 4. Probability Density Functions of irradiance statistics between LOT solution, 2L-WO model and a 10L-WO model when considering only defocus, for an emitted waist of $8 \mathrm{~cm}$.

tures:

$$
\begin{gathered}
\frac{1}{q_{x 0}}=\left(c_{5}^{2}+c_{6}^{2}\right)^{\frac{1}{2}}+\frac{\lambda i}{\pi w_{0}^{2}}, \\
\frac{1}{q_{y 0}}=-\left(c_{5}^{2}+c_{6}^{2}\right)^{\frac{1}{2}}+\frac{\lambda i}{\pi w_{0}^{2}},
\end{gathered}
$$

where $w_{0}$ is the waist size at emission, $\lambda$ is the wavelength and $c_{5,6}$ are the curvatures resulting from astigmatism [10].

Using the ABCD matrix, the complex radius curvatures after propagation through multiple phase screens are ${ }^{1}$ :

$$
q_{x}=\frac{A q_{x 0}+B}{C q_{x 0}+D}
$$

This leads to a modified estimation of the beam radii $w_{x}$ and $w_{y}$ compared to Baker's approach[10]:

$$
w_{(x)}=\sqrt{\frac{\lambda}{\pi \Im\left(1 / q_{(x)}\right)}},
$$

where $\Im$ means the imaginary part. These beam radii are implemented in the LOT solution presented in Equation 19. This permits to obtain the same irradiance estimation as wave optics propagation through multiple screens when only defocus is considered. We call this model WPLOT (for With Propagation Low-Order of Turbulence).

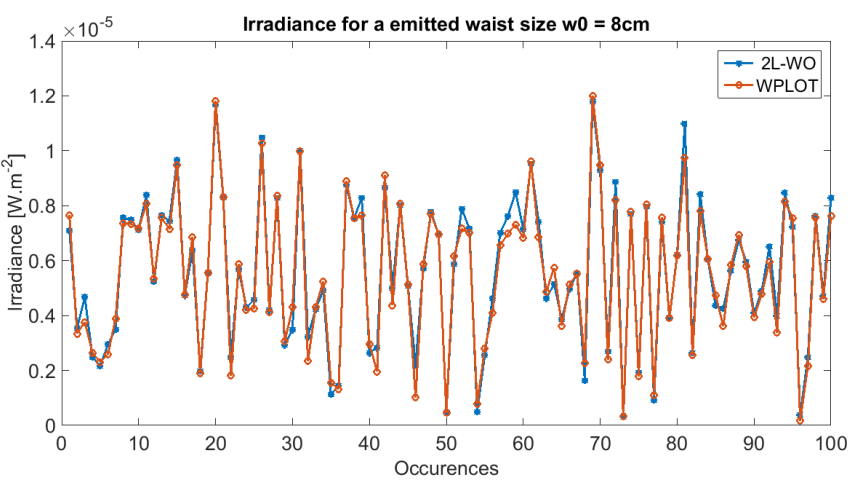

Fig. 5. Random draws of irradiance statistics between WPLOT model (for 2 layer) and the 2L-WO model, for an emitted waist of $8 \mathrm{~cm}$.

\footnotetext{
${ }^{1}$ We present the results in the $x$ direction but the method is exactly the same in the $y$ direction.
}

A comparison between the WPLOT model and the 2L-WO simulation is given in Figure 5. The WPLOT model considers the same two layers as the $2 \mathrm{~L}-\mathrm{WO}$ simulation. We observe that the higher irradiances are much better modeled. For lower irradiances - which are the most interesting ones - the results were already convincing. It is because these lower irradiances are principally due to beam wandering. The remaining errors are due to neglecting the effects of propagation through multiple phase screens for astigmatism. For tilt, the contributions of each layer can be summed while keeping a perfect fit in the far field regardless of the number of atmospheric layers. This approach taking into account the propagation through multiple phase screens does not significantly change the estimation of the irradiance threshold at $5 \%$ of the CDF in the case without tracking. However, it will significantly improve the validity of the results when tracking is taken into account.

\section{A.2. LOT Applicability}

The LOT solution does not take into account Zernike polynomials higher than astigmatism. Using Noll's results for phase variance[14] for a plane wave, the phase variance corresponding to higher orders is

$$
\sigma_{\delta \phi}(j>6)=0.065\left(\frac{d_{0}}{r_{0}}\right)^{\frac{5}{3}},
$$

where $r_{0}$ is the Fried parameter[15], $d_{0}=2 w_{0}$ and $j$ is the number of the considered Zernike polynomial (in our case, all the polynomials of higher order than astigmatism). Statistical equivalence between the model and a case where the whole phase is taken into account is achieved when there is less than 0.1 wave squared difference (within a circle of radius $w_{0}$ ) between the two[10]:

$$
\sigma_{\delta \phi}(j>6) \leq\left(\frac{2 \pi}{10}\right)^{2}
$$

This adds a constraint on the maximum waist size of the beam:

$$
w_{0} \leq 1.5 r_{0} .
$$

This condition ensures that the higher order $(j>6)$ Zernike coefficients' impact on the beam are negligible.

\section{B. Telescope truncation - far field angle}

The model takes into account the parameters of the optical ground station. In particular, one of those parameters is the truncation of the infinite Gaussian beam by the telescope. The truncation will have two effects. The first one is that it will reduce the transmitted power by the telescope [11]. The second one is that it will add a diffraction effect in the far-field. The effects of the diffraction of a Gaussian beam by a circular aperture usually leads to a convolution whose result is not easy to use. Because we consider telescope sizes that will always be larger than the Gaussian beam diameter at emission $\left(2 \times w_{0}\right)$, it is usually considered that the effects of the diffraction of the beam by the aperture will only slightly modify the shape of the beam and in particular, it will become wider while keeping its Gaussian shape. We can use the results from Belland \& al. [16], acquired using energy conservation, to obtain a simple expression of the size of the diffracted waist by the telescope in the case of a propagation without atmospheric turbulence:

$$
w_{L}=\frac{\lambda L}{\pi w_{0}} \frac{\sqrt{1-\exp \left(-\frac{2 R_{T e l}^{2}}{w_{0}^{2}}\right)}}{1-\exp \left(-\frac{R_{T e l}^{2}}{w_{0}^{2}}\right)},
$$


where $R_{T e l}$ is the radius of the telescope. To take into account the truncation effect of the emitter on the beam size, the far-field diffraction expression in Eq.21 is replaced by the waist expression of Eq.9. This leads to a modified expression of the waist in the satellite plane, in the case of Baker's LOT approach:

$$
\begin{aligned}
w_{\{x, y\}}=w_{0} \times[[1 & \left.+L\left(\Delta \kappa \pm \sqrt{c_{5}^{2}+c_{6}^{2}}\right)\right]^{2} \\
+ & {\left.\left[\frac{\lambda L}{\pi w_{0}} \frac{\sqrt{1-\exp \left(-\frac{2 R_{T e l}^{2}}{w_{0}^{2}}\right)}}{1-\exp \left(-\frac{R_{T e l}^{2}}{w_{0}^{2}}\right)}\right]^{2}\right]^{\frac{1}{2}} }
\end{aligned}
$$

This result can lead to an alternate effective beam waist $D_{\text {eff }}$ at $1 / e^{2}$ in irradiance at the emission in order to take into account the effects of the diffraction of the Gaussian beam by a circular aperture. In this paper, we will use Yura's result [17] for the effective beam diameter at $1 / e^{2}$ in irradiance at the emission, which is given by:

$$
D_{e f f}=d_{0}\left[\tanh \left(D_{T e l}^{2} / 2 d_{0}^{2}\right)\right]^{\frac{1}{2}} .
$$

This effective beam diameter yields accurate results well as long as $d_{0}<D_{\text {Tel }}$ (where $d_{0}=2 w_{0}$ ). The encircled power distributions are valid to better than approximately $2 \%$.

However, the strength of the Zernike polynomials coefficients still have to be estimated for a beam of diameter size $d_{0}$ at the emission. Both the methodologies from Yura and Belland\&al. yield similar results. In the rest of the article, we will consider only the methodology from Yura, which is simpler to implement.

The improvement of taking into account the truncation of the beam by the telescope in the WPLOT model can be observed in Figure 6. The results are compared to result from a 2L-WO simulation taking into account a truncation.

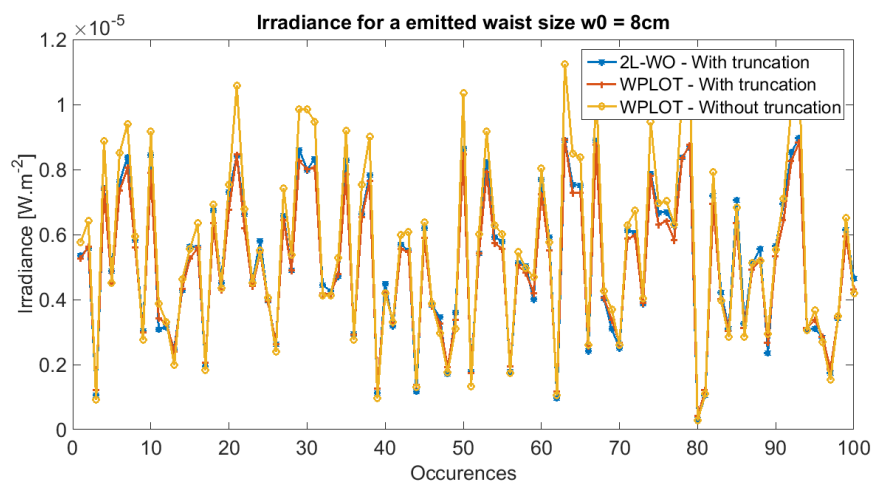

Fig. 6. Random draws of irradiance statistics between WPLOT solution taking into account, or not, the truncation of the Gaussian beam by the telescope compared with 2L-WO simulation taking into account a truncation.

Taking into account the truncation of the Gaussian beam reduces the maximum irradiance that can be reached (at the center of the Gaussian beam) while increasing the width of the beam. This means that at the extremities of the Gaussian beam, when beam wander shifts the Gaussian by a distance equivalent to the beam size, the detected irradiance with a truncated Gaussian beam will be slightly higher than in a no truncation case.
The results show that assuming that the shape of the beam is still Gaussian is relevant.

\section{Presentation of the model process}

There are two aspects to the process of the WPLOT model: the estimation of the Zernike coefficients and the propagation through multiple phase screens. We have shown in Section 3.A.1 that propagation through multiple phase screens essentially impacts defocus and we have proposed a solution to model these effects. For tilt and astigmatism, the turbulence can be integrated and placed at the transmitter prior to propagation.

In a no tracking case, the random draws for tilt and astigmatism can be obtained using the variances given in Eq. 23 and 24 in Appendix C. These variances are estimated over the whole turbulence volume and assume the beam can be modeled as a plane wave. This results from our assumption that the majority of the turbulence is located close to the emitter, where the beam is collimated. If tracking is taken into account, then the estimation of the correlation has to be done over the whole turbulence volume, using multiple layers, because the decorrelation between the downlink and the uplink is usually a function of the altitude. Chassat's correlation functions [18] provide an analytic estimation of the correlation between two beams calculated over the whole turbulence volume and can permit to estimate the tilt contribution of each layer. These contribution can than be integrated and placed at the transmitter prior to propagation.

For defocus, Zernike polynomials have to be estimated for each atmospheric layers. The turbulence strength division between these layers is made to keep the same overall phase variance. In Eq. 24, this implies a change in the estimation of the Fried parameter $r_{0}$, which is now estimated for turbulence volume corresponding to each layer. In our analysis, we will assume that the beam is collimated during its propagation through atmospheric turbulence. This means that we will consider the initial beam diameter $d_{0}$ for each atmospheric layer.

We assume that the Zernike coefficients are Gaussian random variables with zero mean[14], and that they are decorrelated [19].

Times series of each Zernike coefficient can be obtained from their respective temporal Power Spectral Density (PSD). Their PSD are well-known in the literature, especially in the case of plane waves[9] when using Taylor's hypothesis of frozen turbulence, and are given in Eq. 25. The time series of the different Zernike coefficients can be obtained from Eq.12:

$$
a_{i}(t)=\int \sqrt{W_{a_{j}}\left(f_{n}\right)} e^{i \phi_{n}} e^{2 i \pi f_{n} \cdot t} d f_{n},
$$

where $\phi_{n}$ is the phase attributed to the spectral component at the frequency $f_{n}$ and $W_{a_{j}}(f)$ is the temporal PSD of the $a_{j}$ coefficient.

The tilt Zernike coefficients $a_{\{2,3\}}$ are transformed into an angular deviation $\theta_{\{x, y\}}$ using Eq. 27, the defocus Zernike coefficient $a_{4}$ into the defocus curvature $\Delta \kappa$ using Eq. 28 and the Zernike astigmatism coefficients $a_{\{5,6\}}$ into the astigmatism curvatures $c_{\{5,6\}}$ using Eq. 29. The final step is to insert the obtained Zernike coefficients described in Appendix B with the waist solution from Eq. 10.

\section{MODEL VALIDATION}

We have validated our model by comparing its results to wave optics simulations (TURANDOT model[2], dedicated to 
ground to space optical communications). TURANDOT models the propagation of a beam through a realistic atmospheric turbulence. The phase screens are obtained from a von Kármán spectrum and the sampling of the atmospheric turbulence is done using 24 atmospheric layers non uniformly distributed with their locations optimized. For the WPLOT model, we are going to consider the same atmospheric profile as TURANDOT, i.e. 24 layers with the same distribution. Because we focus on the lower irradiances, we need an important number of samples in order to obtain a sufficiently precise of the lower percentages of the irradiance CDFs. However, with TURANDOT, we are limited by the computation time. In our study, we have taken 2000 samples for each case considered with TURANDOT and this led to a computation time of approximately 5 hours each time. On the other hand, with the WPLOT model, we are able to consider a very large number of draws (chosen arbitrarily at 250000 samples) in a approximately 1 minute.

\section{A. Turbulence conditions}

The vertical $C_{n}^{2}$ profile used is the Hufnagel-Valley profile[20] defined by:

$$
\begin{aligned}
C_{n}^{2}(h)=0.00594\left(\frac{v}{27}\right)^{2} & \left(10^{-5} h\right)^{10} e^{-\frac{h}{1000}} \\
& +2.7 \times 10^{-16} e^{-\frac{h}{1500}}+C_{g} e^{-\frac{h}{100}}
\end{aligned}
$$

In this paper, we consider $C_{g}=1.7 \times 10^{-14} \mathrm{~m}^{-\frac{2}{3}}$ and $v=$ $21 \mathrm{~m} / \mathrm{s}$, which results in a $r_{0}$ calculated at zenith equal to $19 \mathrm{~cm}$ for a $1.55 \mu \mathrm{m}$ wavelength. The elevation angle will be considered equal to $45^{\circ}$, making the effective $r_{0}$ equal to $15 \mathrm{~cm}$. The outer scale $L_{0}$ is fixed by the wave optics simulations: we have taken the outer scale to be equal to TURANDOT's phase screens' sizes, which gives $L_{0}=2.5 \mathrm{~m}$ in our case.

The temporal effects are modeled using Taylor's hypothesis of frozen turbulence and a Bufton wind profile[20], described in Equation 14, in which the atmospheric layers move with a $5 \mathrm{~m} / \mathrm{s}$ speed at ground level and with a $30 \mathrm{~m} / \mathrm{s}$ speed at an altitude around $12.5 \mathrm{~km}$ :

$$
V(h)=S_{G}+S_{P} e^{-\left(\frac{h-H_{P}}{W_{P}}\right)^{2}},
$$

in which we have taken $S_{G}=5 \mathrm{~m} / \mathrm{s}, S_{P}=25 \mathrm{~m} / \mathrm{s}, H_{P}=$ $12448 \mathrm{~m}$ and $W_{P}=4800 \mathrm{~m}$.

We will consider only the case in which the wind moves in the $x$ direction for all layers. Extension to the wind moving in multiple direction is straightforward $([9,18])$.

Using these atmospheric conditions and Baker's conditions on $N_{L}$ and $N_{\text {tau }}$ presented in Eq.18 to define the $D_{1}$ region lead to a minimum waist size of $4.9 \mathrm{~cm}$ (and a maximum waist size of $4.2 \mathrm{~m}$ but our assumptions on phase variance already impose the maximum bound $w_{0} \leq 1.5 r_{0}=22.5 \mathrm{~cm}$ presented in Eq.8).

\section{B. Average irradiance and scintillation index}

In this example, we will consider the telescope diameter $D_{T_{X}}=$ $2^{\frac{3}{2}} w_{0}$, which is a value often proposed in the literature[21]. It optimizes the losses due to beam truncation and beam diffraction. We have compared the mean detected on-axis irradiance (Fig.7) and on-axis scintillation index $\sigma_{I}^{2}$ (Fig.8) obtained with wave optics simulations and our model as a function of the waist size at emission.

There is good agreement between both TURANDOT and WPLOT model, which tends to validate WPLOT model.

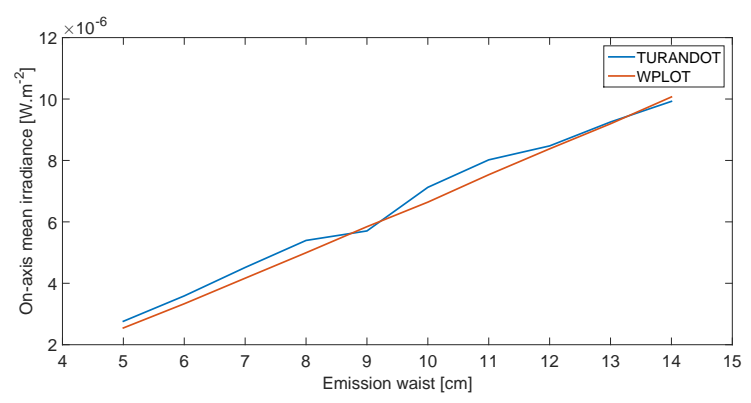

Fig. 7. Mean detected on-axis irradiance as a function of the emitted waist size. The mean on-axis irradiance has been calculated over 2000 samples for the TURANDOT simulations and over 250000 samples for the WPLOT model, for waist sizes ranging from 5 to $14 \mathrm{~cm}$.

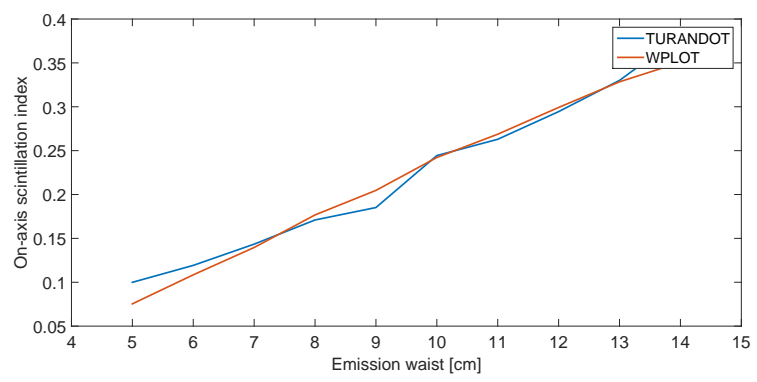

Fig. 8. On-axis scintillation index as a function of the emitted waist size. The on-axis scintillation index has been calculated for over 2000 samples for the TURANDOT simulations and over 250000 samples for the WPLOT model, for waist sizes ranging from 5 to $14 \mathrm{~cm}$.

\section{Cumulative Density Function}

We also compare the Cumulative Density Functions (CDF) obtained with TURANDOT simulations and WPLOT model. Some examples are given in Figures 9, 10, 11 and 12 for waist sizes of $5,8,11$ and $14 \mathrm{~cm}$.

There are some differences between our model and the results coming from TURANDOT. However, there is a good fit for the lower irradiances, which are the interesting parameter for our study. The threshold at $5 \%$ of the CDF for different waist sizes at the emission is given in Figure 13.

We observe a very good fit for the smaller waists but a gap starts to appear for waist sizes bigger than $9 \mathrm{~cm}$. The error on the estimation of the threshold starts to be bigger than $1 \mathrm{~dB}$ for waists bigger than $12 \mathrm{~cm}$. There can be a multiple reasons for this gap. It might mean that the impact of higher order phase effects, such as spherical aberration or coma for example, which are not taken into account, are no longer negligible. This would mean that the condition from Equation 8 may not be sufficiently strong.

N.B.: The envisioned diameters for the emitting telescopes will probably not be bigger than $40 \mathrm{~cm}$. Therefore, a waist size of $14 \mathrm{~cm}$ is the maximum that needs to be considered in a case where the emitting telescope diameter $D_{T_{X}}$ is equal to $2^{\frac{3}{2}} w_{0}$.

\section{Temporal Power Spectral Density}

We have also compared the on-axis irradiance temporal PSD obtained with TURANDOT and the WPLOT model. These temporal PSDs are obtained from time series estimated over a du- 


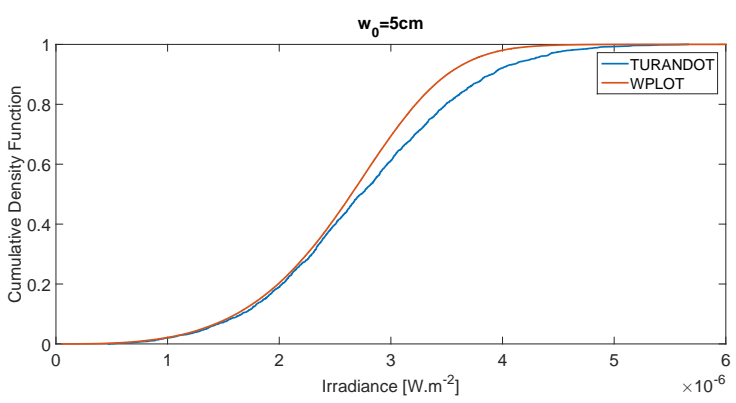

Fig. 9. Cumulative density function of the detected on-axis irradiance comparison between the TURANDOT simulation and the WPLOT model for a waist size of $5 \mathrm{~cm}$. The CDFs have been calculated over 2000 samples for the TURANDOT simulation and over 250000 samples for the WPLOT model.

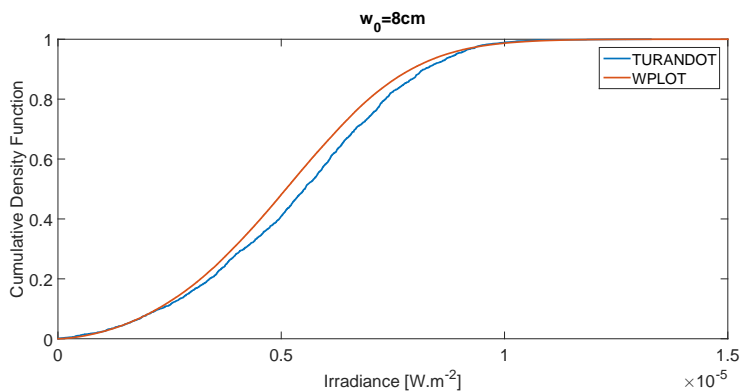

Fig. 10. Cumulative density function of the detected on-axis irradiance comparison between the TURANDOT simulation and the WPLOT model for a waist size of $8 \mathrm{~cm}$. The CDFs have been calculated over 2000 samples for the TURANDOT simulation and over 250000 samples for the WPLOT model.

ration of $4 \mathrm{~s}$ with a $2500 \mathrm{~Hz}$ sampling. Some examples are given in Figures 14 and 15 for waist sizes of 8 and $14 \mathrm{~cm}$.

Both figures show good agreement between the WPLOT model and TURANDOT ${ }^{2}$. As the WPLOT model is much faster than TURANDOT, it is able to create much longer time series in reasonable time. For example, the 4 second time series with a $2500 \mathrm{~Hz}$ sampling considered were obtained in a few seconds with the WPLOT model whereas it took around a day with TURANDOT. Because we have shown that we have a precise estimation of the irradiance threshold at $5 \%$ of the irradiance CDF (cf. Figure 13), the fact that we can obtain very long time series means that the WPLOT model can be used to study the fade statistics.

\section{E. Justification of model particularities}

\section{E.1. Impact of taking into account the beam truncation}

Most models found in the literature do not take into account the impact of the beam truncation $([5,8,21])$. However, we show that its influence is not always negligible and it is therefore an important parameter to take into account if the objective is to optimize the architecture of an optical ground station. In Figure 16 , we use the scintillation index as an indicator of the influence of the beam truncation as a function of the emitting waist size. The beam truncation is $D_{T_{X}}=2^{\frac{3}{2}} w_{0}$.

${ }^{2}$ We observe a floor on the temporal PSD obtained with TURANDOT, which resembles aliasing effects. It would mean that the phase screen sampling of TURANDOT is insufficient. However, since these effects appear after the cutoff frequency, we have not look further into this subject.

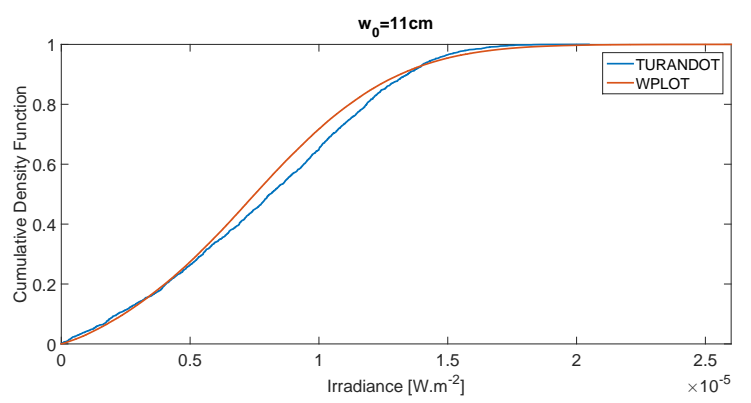

Fig. 11. Cumulative density function of the detected on-axis irradiance comparison between the TURANDOT simulation and the WPLOT model for a waist size of $11 \mathrm{~cm}$. The CDFs have been calculated for over 2000 samples for the TURANDOT simulation and over 250000 samples for the WPLOT model.

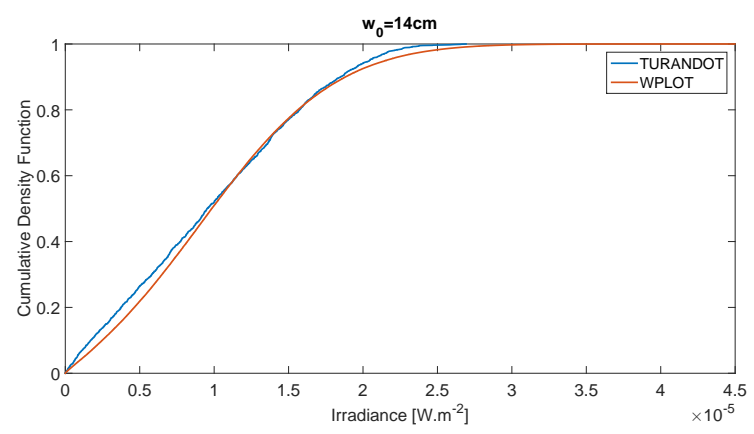

Fig. 12. Cumulative density function of the detected on-axis irradiance comparison comparison between the TURANDOT simulation and the WPLOT model for a waist size of $14 \mathrm{~cm}$. The CDFs have been calculated for over 2000 samples for the TURANDOT simulation and over 250000 samples for the WPLOT model.

We therefore observe that, if the beam truncation is not taken into account, there is an overestimation of the scintillation index. This is because the waist size at satellite level is smaller. Therefore, beam wander induces more important irradiance fluctuations.

\section{E.2. Comparison of WPLOT model to Rytov Perturbation Method}

We justify here our choice of Baker's model[10] as base for our model by comparing it with other usual models in the literature where the defocus and the astigmatism are not taken into account and rely for the higher orders on the Rytov approximation $([5,8,21])$. In Figure 17, we compare the scintillation results from the LOT model to results obtained from Dios' methodology[8]. In order to take into account the truncation, we have taken the waists obtained from Yura (see Eq.11).

We observe that results from Dios slightly overestimate the scintillation index compared to TURANDOT and the WPLOT model. This proves that the effects of defocus and astigmatism can't be neglected. Moreover, using the Rytov perturbation method does not permit to compensate the effects of defocus and astigmatism on irradiance fluctuations.

\section{MODEL APPLICATION TO TRACKING}

Taking into account tracking within the model is pretty straightforward using the literature $([9,18])$. The important results are 


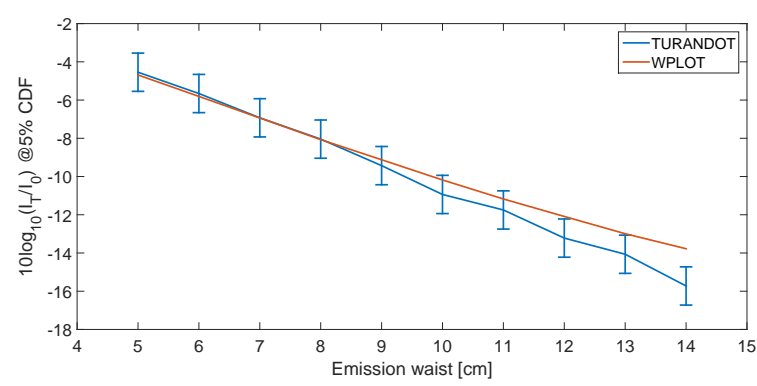

Fig. 13. Comparison of the estimation of the threshold at $5 \%$ of the CDF between the TURANDOT simulations and the WPLOT model for different waist sizes at the emission. The bars correspond to a $1 \mathrm{~dB}$ margin from TURANDOT's results.

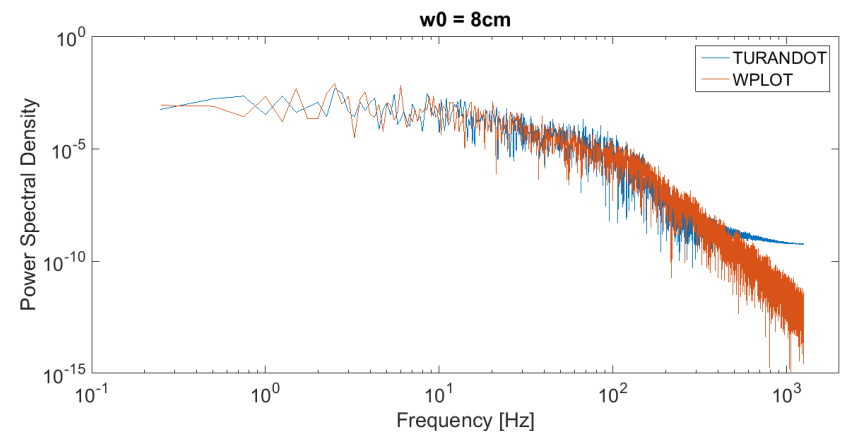

Fig. 14. Temporal power spectral density of on-axis irradiance comparison for a waist size of $8 \mathrm{~cm}$ calculated for an irradiance time series of $4 \mathrm{~s}$ with $2500 \mathrm{~Hz}$ sampling.

presented in Appendix D.

It is now possible to compare the statistical results as well as the time series when tracking is added. We present in this Section the results obtained for a specific example in which the point-ahead angle is considered equal to $18.5 \mu \mathrm{rad}$. The delay between the measurement on the downlink and the precompensation of the uplink is considered equal to $4 \mathrm{~ms}$. Therefore, tracking will not be perfect. The pupils of downlink and uplink are merged at ground level. The atmospheric parameters are the same as in Section 4.

While the limitations on the quality of the correction due to the point-ahead angle will always be present, the limitations due to the delay can easily be dealt with by increasing the bandwidth of the correction loop. In the considered case, the tilt correlation obtained from Chassat's functions is equal to 0.86 for a waist size of $8 \mathrm{~cm}$. This leads to a residual tilt variance of 0.03 $\mathrm{rad}^{-2}$, which then leads to a standard deviation of the displacement in the satellite plane of $38 \mathrm{~m}$. If the delay before the correction is zero, then the tilt correlation becomes 0.9 , the residual tilt variance becomes $0.02 \mathrm{rad}^{-2}$ and the standard deviation of the displacement is $33 \mathrm{~m}$. Compared to the beam radius in the satellite plane, equal to $254 \mathrm{~m}$, this $5 \mathrm{~m}$ displacement error is negligible, which means that there is no need for a lower delay time.

\section{A. Validation}

We have compared the results obtained with the WPLOT model and TURANDOT when taking into account tracking. Figures 18 shows the estimation of the threshold at $5 \%$ of the CDF between TURANDOT and the WPLOT model for different waist

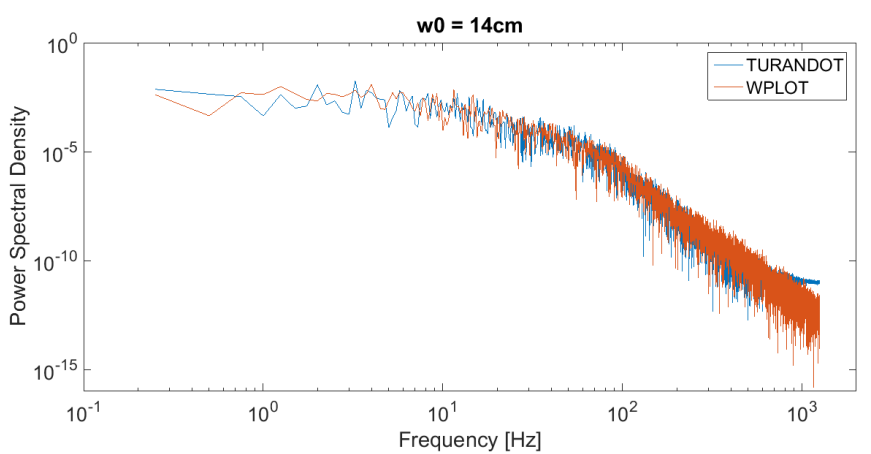

Fig. 15. Temporal power spectral density of on-axis irradiance comparison for a waist size of $14 \mathrm{~cm}$ calculated for an irradiance time series of $4 \mathrm{~s}$ with $2500 \mathrm{~Hz}$ sampling.

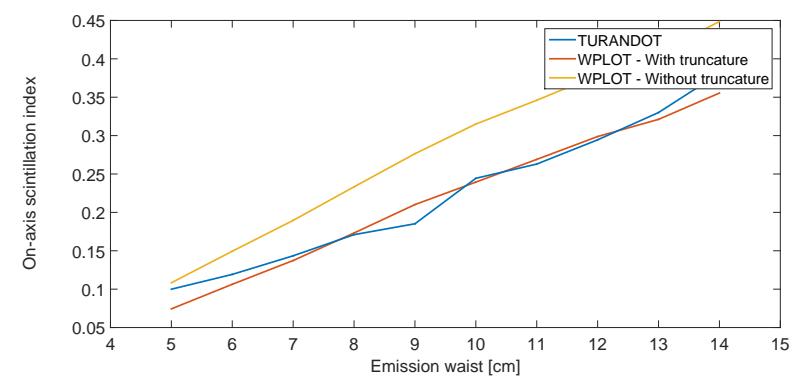

Fig. 16. Impact of not taking into account the beam truncation on the on-axis scintillation index, for waist sizes ranging from 5 to $14 \mathrm{~cm}$ and estimated over 250000 samples.

sizes at emission. This shows that the estimation of the threshold is with a precision lower than $1 \mathrm{~dB}$. Figure 19 shows the temporal PSDs of irradiance fluctuations for the specific case of a $8 \mathrm{~cm}$ waist size at emission obtained with TURANDOT and the WPLOT model. We observe that there is a good match.

\section{B. Improvement on link budget}

We have compared the mean detected irradiance (Fig.20) and scintillation index $\sigma_{I}^{2}$ (Fig.21) with and without tracking as a function of the waist size at emission, using the WPLOT model. In this section, we will consider two layers.

The results are consistent with what one would expect: tracking the tip/tilt significantly reduces the scintillation and increases the mean irradiance. These effects are stronger for the larger waist sizes at the emission than for the lower waist sizes.

We can also observe the evolution of time series with and without tracking in Figure 22. With tracking, we observe the disappearance of the very low irradiance fadings. This means that, if we keep the irradiance threshold at $5 \%$ of the CDF, the fading times are going to be shorter, but more numerous. It also shows the significant increase of the irradiance threshold at $5 \%$ of the cumulative density function and therefore the important decrease of the losses due to turbulence $L_{T U R B}$, as can be seen in Figure 23.

\section{CONCLUSION}

We have presented a model which performs time series of the irradiance fluctuations induced by the atmospheric turbulence on the axis in the satellite plane in case of uplink. Moreover, this 


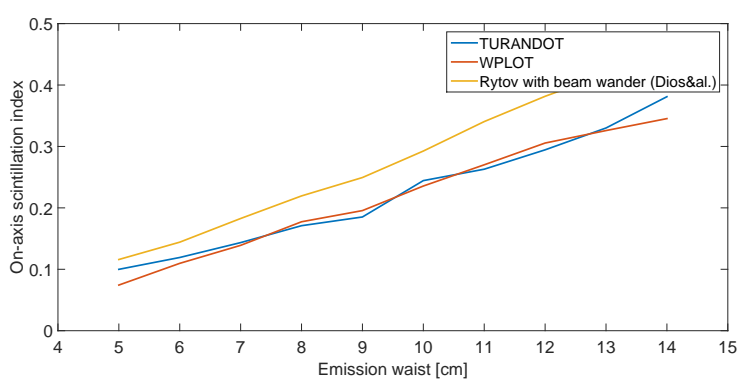

Fig. 17. Impact of not taking into account the defocus and astigmatism on the on-axis scintillation index and estimated over 250000 samples.

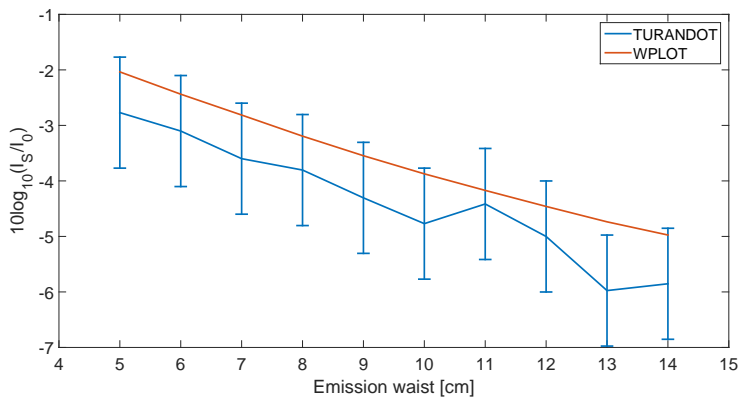

Fig. 18. Comparison of the estimation of the threshold at $5 \%$ of the CDF between the TURANDOT simulation and the WPLOT model for different waist sizes at the emission. The bars correspond to $1 \mathrm{~dB}$ margin from TURANDOT's results.

model is directly linked to the optical ground station characteristics and turbulence profile. Compared to wave optics simulation (TURANDOT), the computation time is considerably reduced (approximately 5 hours to obtain 2000 samples using TURANDOT while the WPLOT model provides the results for 250000 samples in less than a minute). This allows sensitivity studies and longer time series generation.

Because we focus on a ground to satellite propagation case, we have been able to make justified hypotheses that significantly simplify our model by restricting its range of validity to telescope diameters between $10 \mathrm{~cm}$ and $40 \mathrm{~cm}$. This model has been validated by comparing it to wave optics simulations.

The model takes into account the different parameters of the optical ground station architecture. So we are able to evaluate the optical link budget as a function of them and optimize the performance of the ground station. Besides, different errors can be considered for the correction (point-ahead, angle, servoloop delay, and even pupil misalignment between the measuring pupil of the downlink and the emitting pupil of the uplink) as well as effects due to the finite telescope diameter on the infinite gaussian beam. These effects are not usually taken into account in the literature $[8,21]$.

Contrary to many works in the literature $([4,5,22])$, which focus on the mean fade duration and mean number of fades per second, our model can create time series of the irradiance fluctuations. This approach will be useful to work on the contributions of interleaving and on the error correction codes. Despite some discrepancies that appear for the greater irradiances, the time series obtained with the WPLOT model enable a precise study of the fades as they occur in the lower irradiances which

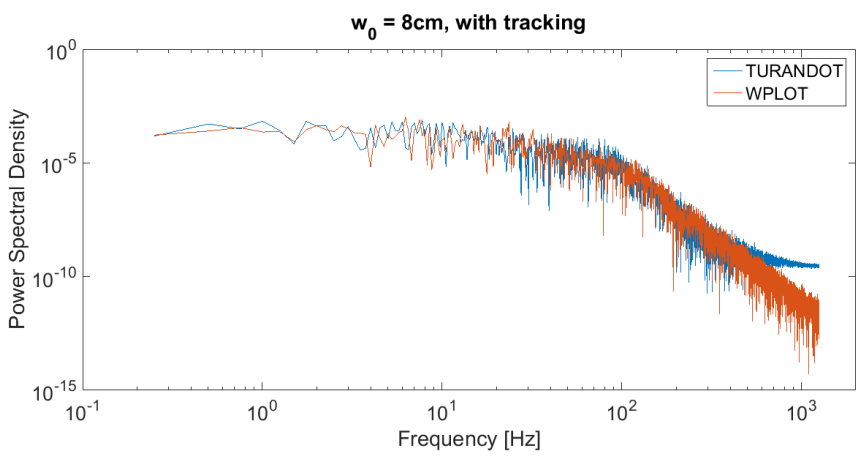

Fig. 19. Comparison of the on-axis irradiance temporal PSD between the TURANDOT simulation and the WPLOT model with tracking taken into account for a $8 \mathrm{~cm}$ waist size at emission calculated for an irradiance time series of $4 \mathrm{~s}$ with 10000 samples.

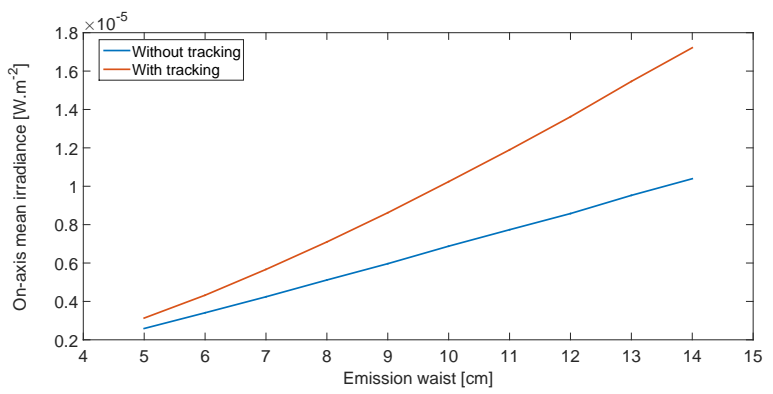

Fig. 20. Comparison of the mean detected on-axis irradiance as a function of the emitted waist size calculated over 250000 samples with and without tracking, using the WPLOT model with 2 layers.

are well modeled. The WPLOT model therefore provides the possibility to estimate the fade duration occurrence probability and the cumulative fade duration exceedance probability as defined in [23] from the International Telecommunication Union (ITU) recommendations [24].

Some work has already been done on the optimization of an optical ground station using this model [25]. The time series provided will be useful for future work, notably the optimization of interleaving and error correction codes in the presence of turbulence.

\section{A. MODELS DESCRIPTION}

Throughout this article, we use multiple models, which are presented in Table 1 for improved clarity.

\section{B. LOT IRRADIANCE MODEL PRESENTATION}

\section{Hypotheses and range of validity}

In order to determine the range of validity, Baker[10] proposes two dimensionless parameters to define the region of interest in which beam wander becomes predominant. These parameters are

$$
\begin{aligned}
& N_{L}=\frac{\pi w_{0}^{2}}{\lambda L}, \\
& N_{\tau}=\frac{\pi w_{0}^{2}}{\lambda z_{\tau}},
\end{aligned}
$$




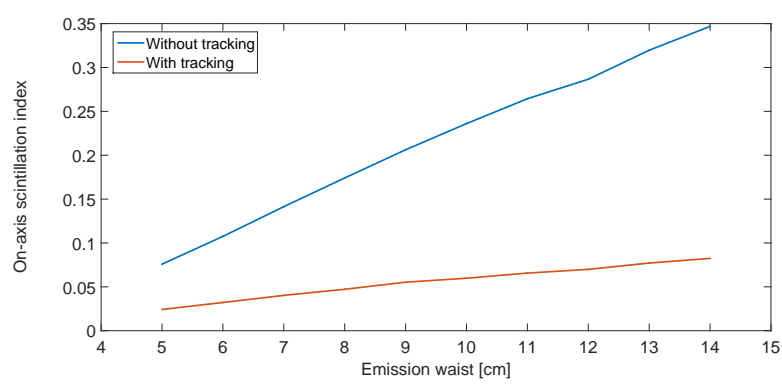

Fig. 21. Comparison of the on-axis scintillation index as a function of the emitted waist size calculated over 250000 samples with and without tracking, using the WPLOT model with 2 layers.

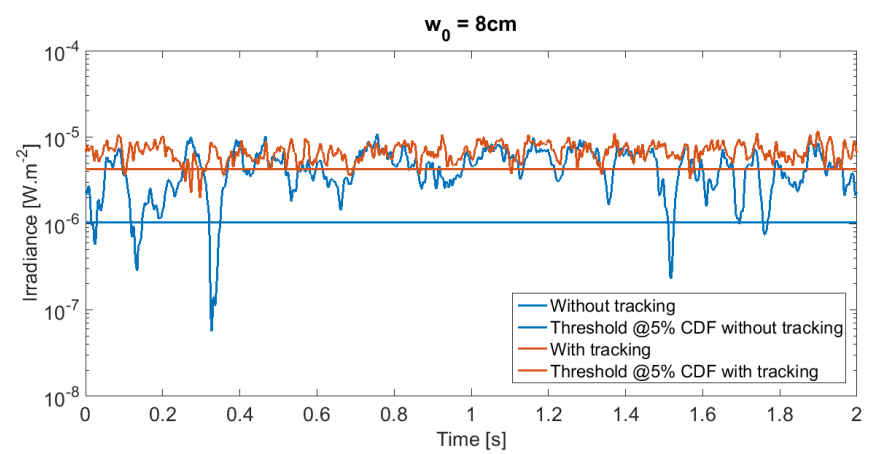

Fig. 22. $2 \mathrm{~s}$ on-axis irradiance time series with a $2500 \mathrm{~Hz}$ sampling for a waist size of $8 \mathrm{~cm}$, obtained with the WPLOT model with and without tracking.

with $z_{\tau}$ defined by

$$
z_{\tau}=\frac{\int_{0}^{L} z C_{n}^{2}(z) d z}{\int_{0}^{L} C_{n}^{2}(z) d z}
$$

$w_{0}$ is the waist radius at $1 / e$ of the amplitude, $\lambda$ is the wavelength, $L$ is the distance between the ground and the satellite and $C_{n}^{2}$ is the refractive index structure constant, corresponding to the variance of the refractive index between two points separated between $1 \mathrm{~m} . z_{\tau}$ gives an estimation of the centroid of the turbulence strength on the path. The shorter it is, the stronger is the turbulence near the emitter. The parameters $N_{L}$ and $N_{\tau}$ correspond to Fresnel numbers of the initial beam observed at, respectively, distance $L$ and $z_{\tau}$. They compare the Rayleigh range of the beam to, respectively, the distances $L$ and $z_{\tau}$. According to Baker, the bounds of this region are given by:

$$
N_{L}<1 \quad \& \quad N_{\tau}^{2} \gg 1 \text {. }
$$

\section{Low-Order Turbulence solution}

Baker[10] proposes a solution to describe the irradiance fluctuations relying on an approach that describes the deformations of a Gaussian beam propagating through the atmosphere as dependent on the first and second order Zernike polynomials [12],[13]: tip/tilt, defocus and astigmatism. He has shown that the resulting irradiance of a Gaussian beam, of wavelength $\lambda$ (and wavenumber $k=\frac{2 \pi}{\lambda}$ ) and of waist size $w_{0}$ (at $1 / e^{2}$ of the Gaussian irradiance) at the emission, propagating through

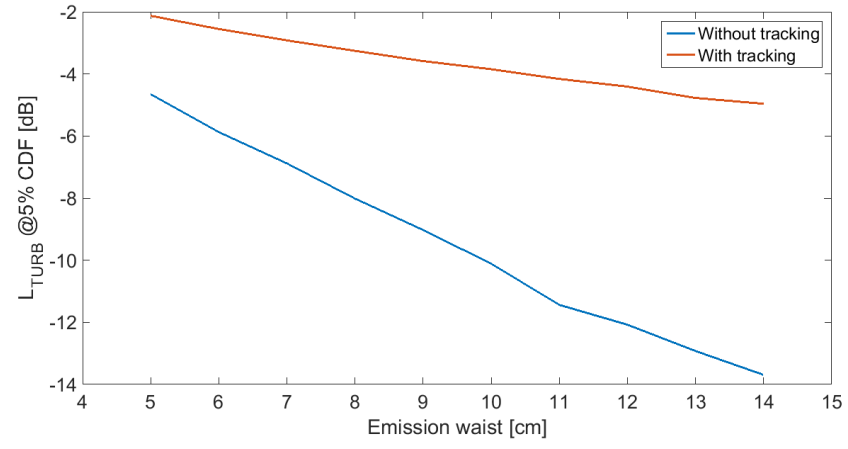

Fig. 23. $L_{T U R B}$ estimation with and without tracking for different emitted waist sizes, obtained using the WPLOT model using 250000 samples.

atmospheric turbulence up to a satellite at a distance $L$ is expressed

$$
\begin{aligned}
& I(x, y, L)=\frac{2}{\pi} \frac{1}{w_{x} w_{y}} \\
& \quad \times \exp \left(-\frac{2}{w_{x}^{2}}\left[\left(x-\delta_{x}\right) \cos (\omega)+\left(y-\delta_{y}\right) \sin (\omega)\right]^{2}\right) \\
& \times \exp \left(-\frac{2}{w_{y}^{2}}\left[\left(x-\delta_{x}\right) \sin (\omega)-\left(y-\delta_{y}\right) \cos (\omega)\right]^{2}\right),
\end{aligned}
$$

where the beam wander, partial beam radii, and astigmatism parameters in Eq.19 are given by, respectively,

$$
\delta_{\{x, y\}}=L \theta_{\{x, y\}}
$$

$$
w_{\{x, y\}}=w_{0} \sqrt{\left[1+L\left(\Delta \kappa \pm \sqrt{c_{5}^{2}+c_{6}^{2}}\right)\right]^{2}+\left(\frac{2 L}{k w_{0}^{2}}\right)^{2}},
$$

and

$$
\omega=\frac{1}{2} \arg \left(c_{5}+j c_{6}\right) .
$$

$\theta_{\{x, y\}}$ corresponds to the angular tip/tilt in radians. $\Delta \kappa$ corresponds to the defocus curvature and $c_{\{5,6\}}$ to the astigmatism curvature (given in $m^{-1}$ ).

In Eq.21, the first term under the root square expresses the broadening of the beam induced by defocus and astigmatism and the second term is the broadening induced by diffraction.

\section{ZERNIKE COEFFICIENTS}

\section{Variances}

The variances of the Zernike coefficients are very well known in the literature[18] in the case of a von Kármán spectrum for atmospheric turbulence. The variance for tip and tilt, of radial order $n=1$, is

$$
\begin{gathered}
\sigma_{a_{2,3}}^{2}=0.451\left(\frac{d_{0}}{r_{0}}\right)^{\frac{5}{3}} \\
\times\left[1-0.77\left(\frac{2 \pi d_{0}}{L_{0}}\right)^{\frac{1}{3}}+0.09\left(\frac{2 \pi d_{0}}{L_{0}}\right)^{2}-0.054\left(\frac{2 \pi d_{0}}{L_{0}}\right)^{\frac{7}{3}}\right],
\end{gathered}
$$




\begin{tabular}{|c|c|c|}
\hline Model & $\begin{array}{l}\text { Phase screens dis- } \\
\text { tribution }\end{array}$ & $\begin{array}{l}\text { Propagation } \\
\text { method }\end{array}$ \\
\hline LOT & $\begin{array}{l}1 \text { phase screen } \\
\text { obtained from } \\
\text { the linear combi- } \\
\text { nation of tip/tilt, } \\
\text { defocus and } \\
\text { astigmatism. } \\
\text { It is placed at } \\
\text { transmitter. }\end{array}$ & $\begin{array}{l}\text { Analytic propaga- } \\
\text { tion of Gaussian } \\
\text { beam ( } w_{x} \text { and } w_{y} \\
\text { estimation). }\end{array}$ \\
\hline WPLOT & $\begin{array}{l}\text { Multiple phase } \\
\text { screens obtained } \\
\text { from the linear } \\
\text { combination of } \\
\text { tip/tilt, defocus } \\
\text { and astigmatism. } \\
\text { They are dis- } \\
\text { tributed along the } \\
\text { propagation path. }\end{array}$ & $\begin{array}{l}\text { Analytic propa- } \\
\text { gation of defocus } \\
\text { leading to another } \\
\text { estimation of } w_{x} \\
\text { and } w_{y} .\end{array}$ \\
\hline 2L-WO & $\begin{array}{l}2 \text { phase screens } \\
\text { obtained from } \\
\text { the linear combi- } \\
\text { nation of tip/tilt, } \\
\text { defocus and astig- } \\
\text { matism (one at } \\
\text { ground layer and } \\
\text { one at a } 10 \mathrm{~km} \\
\text { altitude) }\end{array}$ & $\begin{array}{l}\text { Fresnel propaga- } \\
\text { tion between the } \\
\text { phase screens and } \\
\text { up to the satellite }\end{array}$ \\
\hline TURANDOT & $\begin{array}{l}\text { Multiple phase } \\
\text { screens obtained } \\
\text { from a von Kár- } \\
\text { mán spectrum } \\
\text { and distributed } \\
\text { along the propa- } \\
\text { gation path }\end{array}$ & $\begin{array}{l}\text { Fresnel propaga- } \\
\text { tion between the } \\
\text { phase screens and } \\
\text { up to the satellite }\end{array}$ \\
\hline
\end{tabular}

Table 1. Models description.

where $L_{0}$ is the outer scale. The defocus and astigmatism Zernike coefficients variances, of radial order $n=2$, are

$$
\begin{aligned}
\sigma_{a_{4,5,6}}^{2}=2.34 & \times 10^{-2}\left(\frac{d_{0}}{r_{0}}\right)^{\frac{5}{3}} \\
& \times\left[1-0.39\left(\frac{2 \pi d_{0}}{L_{0}}\right)^{2}+0.27\left(\frac{2 \pi d_{0}}{L_{0}}\right)^{\frac{7}{3}}\right] .
\end{aligned}
$$

N.B.: In the case of the WPLOT model, $\sigma_{a_{4}}^{2}$ has to be estimated for each layer. This means that the Fried parameter $r_{0}$ in Eq.24 has to be calculated between the bounds of each atmospheric layer.

\section{Power Spectral Densities}

The PSDs for each Zernike coefficient are [9]:

$$
\begin{aligned}
& W_{a_{j}}(v)= \\
& \int_{0}^{L} \int_{-\infty}^{\infty} \frac{1}{V(z)}\left|\tilde{M}_{a_{j}}\left(\frac{v}{V(z)}, f_{y}\right)\right|^{2} W_{\phi}\left(\frac{v}{V(z)}, f_{y}\right) d z d f_{y}
\end{aligned}
$$

where $W_{\phi}$ is the phase spatial power spectrum and $\left|\tilde{M}_{a_{j}}(\vec{f})\right|$ is the Fourier transform of the Zernike polynomial:

$$
\begin{aligned}
\left|\tilde{M}_{a_{j}}(\vec{f})\right| & =\sqrt{n+1} \frac{2\left|J_{n+1}\left(\pi d_{0} f\right)\right|}{\pi d_{0} f} \\
& \times\left\{\begin{array}{lc}
\sqrt{2}|\cos (m \theta)| & \text { for } m \neq 0 \text { \& } j \text { even } \\
\sqrt{2}|\sin (m \theta)| & \text { for } m \neq 0 \text { \& } j \text { odd }, \\
1 & \text { for } m=0
\end{array}\right.
\end{aligned}
$$

where $\vec{f}=\left(f_{x}, f_{y}\right)$ when the frequency vector is described in cartesian coordinates or $\vec{f}=(f, \theta)$ when it is described in polar coordinates, $n$ is the radial degree and $m$ the azimuthal frequency of the $j^{\text {th }}$ Zernike polynomial. These results are for a plane wave.

\section{From Zernike coefficients to phase coefficients}

Once the Zernike coefficients are obtained, they can easily be converted into the corresponding phase coefficients in order to insert them in the model.

The angular deviation due to tip/tilt is

$$
\theta_{x, y}=\left(\frac{2 \lambda}{\pi d_{0}}\right) a_{2,3}
$$

while the defocus curvature is

$$
\Delta \kappa=\left(\frac{8 \sqrt{3}}{\pi}\right)\left(\frac{\lambda}{d_{0}^{2}}\right) a_{4},
$$

and the astigmatism curvature is

$$
c_{5,6}=\left(\frac{4 \sqrt{6}}{\pi}\right)\left(\frac{\lambda}{d_{0}^{2}}\right) a_{5,6} .
$$

\section{TAKING INTO ACCOUNT TRACKING}

The idea is to inject the opposite of the Zernike tip/tilt coefficients $\left(a_{2} / a_{3}\right)$ measured on the downlink to pre-compensate the uplink. The pointing error at the exit of the atmosphere is

$$
a_{i, r e s}(t)=a_{i, U}(t)-a_{i, D}(t),
$$

where $a_{i, r e s}, a_{i, U}(t)$ and $a_{i, D}(t)$ are respectively the residual tip/tilt error $(i=2,3)$, the tip/tilt on the uplink without precompensation and the tip/tilt measured on the downlink.

\section{A. Reciprocity principle and implications}

The idea of compensation relies on the reciprocity of light propagation. However, a difficulty is that, while the downlink is a plane wave, the uplink is a Gaussian beam. It has been shown $([26],[27])$ that the upward propagation of a Gaussian beam can be modeled by the downward propagation of a plane wave. This result simplifies the estimation of the correlation between the downlink and the uplink. It justifies our use of the Zernike polynomials compared to Laguerre polynomials that used in the case of a Gaussian beam. It is also consistent with the assumption that the beam is collimated throughout its propagation in the atmosphere. 


\section{B. Statistical approach}

The variance of the residual Zernike coefficient is

$$
\sigma_{a_{i, \text { res }}}^{2}=\left\langle\left(a_{i, U}-a_{i, D}\right)^{2}\right\rangle \text {. }
$$

This leads to

$$
\sigma_{a_{i, r e s}}^{2}=\left\langle a_{i, U}^{2}\right\rangle+\left\langle a_{i, D}^{2}\right\rangle-2\left\langle a_{i, U} a_{i, D}\right\rangle,
$$

where $\left\langle a_{i, U} a_{i, D}\right\rangle$ is the covariance between the $i^{\text {th }}$ Zernike coefficients of the uplink and the downlink. An estimation is given in F. Chassat's thesis[18].

\section{Power Spectral Densities after tracking correction}

In the case of tracking, the results are obtained from the differential phase. Assuming the on-ground pupil is the same diameter, we obtain the phase related quantity G[9]:

$$
G(\vec{r})=[\phi(\vec{r}+\vec{D}, t)-\phi(\vec{r}, t)] * Z_{j}(\vec{r}),
$$

where $Z_{j}(\vec{r})$ is the $j^{\text {th }}$ Zernike polynomial defined on a pupil of diameter $d_{0}$.

If we consider that $\vec{D}$ is oriented along the $x$ axis, the spectrum from Equation 25 becomes

$$
\left|\tilde{M}_{G}(\vec{f}, h)\right|^{2}=4 \sin ^{2}\left(\pi D \frac{v}{V(h)}\right) \times\left|\tilde{M}_{a_{j}}(\vec{f})\right|^{2},
$$

where $\left|\tilde{M}_{a_{j}}(\vec{f})\right|$ is obtained from Equation 26.

Acknowledgments The authors would like to thank JeanMarc Conan and Nicolas Védrenne for many helpful discussions.

\section{REFERENCES}

1. D. L. Fried and H. T. Yura, "Telescope-performance reciprocity for propagation in a turbulent medium," J. Opt. Soc. Am. 62, 600-602 (1972).

2. N. Védrenne, J. Conan, M. Velluet, M. Sechaud, M. Toyoshima, H. Takenaka, A. Guérin, and F. Lacoste, "Turbulence effects on bi-directional ground-to-satellite laser communication systems," in "Proc. International Conference on Space Optical Systems and Applications," (2012).

3. J. M. Martin and S. M. Flatté, "Intensity images and statistics from numerical simulation of wave propagation in 3-d random media," Appl. Opt. 27, 2111-2126 (1988).

4. L. C. Andrews and R. L. Phillips, Laser beam propagation through random media, vol. 1 (SPIE press Bellingham, WA, 2005).

5. S. Basu, D. Voelz, and D. K. Borah, "Fade statistics of a ground-tosatellite optical link in the presence of lead-ahead and aperture mismatch," Applied Optics 48, 1274 to 1287 (2009).

6. L. C. Andrews, R. L. Phillips, and P. T. Yu, "Optical scintillations and fade statistics for a satellite-communication system," Appl. Opt. 34, 77427751 (1995).

7. G. J. Baker and R. S. Benson, "Gaussian-beam weak scintillation on ground-to-space paths: compact descriptions and rytov-method applicability," Optical Engineering 44, 106002-106002 (2005).

8. F. Dios, J. A. Rubio, A. Rodríguez, and A. Comerón, "Scintillation and beam-wander analysis in an optical ground station-satellite uplink," Appl. Opt. 43, 3866-3873 (2004).

9. J.-M. Conan, G. Rousset, and P.-Y. Madec, "Wave-front temporal spectra in high-resolution imaging through turbulence," J. Opt. Soc. Am. A 12, 1559-1570 (1995).

10. G. J. Baker, "Gaussian beam weak scintillation: low-order turbulence effects and applicability of the rytov method," J. Opt. Soc. Am. A 23 , 395-417 (2006).
11. B. J. Klein and J. J. Degnan, "Optical antenna gain. 1: Transmitting antennas," Appl. Opt. 13, 2134-2141 (1974).

12. F. Zernike, "Diffraction theory of the knife-edge test and its improved form, the phase-contrast method," Monthly Notices of the Royal Astronomical Society 94, 377-384 (1934).

13. M. Born and E. Wolf, "Principles of optics: electromagnetic theory of propagation, interference and diffraction of light," (1975).

14. R. J. Noll, "Zernike polynomials and atmospheric turbulence," Journal of the Optical Society of America 66, 207-211 (1976).

15. D. Fried, "Optical resolution through a randomly inhomogenous medium for very long and very short exposures," Journal of the Optical Society of America pp. 1372-1379 (1966)

16. P. Belland and J. P. Crenn, "Changes in the characteristics of a gaussian beam weakly diffracted by a circular aperture," Appl. Opt. 21, 522527 (1982).

17. H. T. Yura, "Optimum truncation of a gaussian beam for propagation through atmospheric turbulence," Appl. Opt. 34, 2774-2779 (1995).

18. F. Chassat, "Propagation optique à travers la turbulence atmosphérique : étude modale de l'anisoplanétisme et application à l'optique adaptative," Physique, Université Paris-Sud (1992).

19. N. A. Roddier, "Atmospheric wavefront simulation using zernike polynomials," Optical Engineering 29, 1174-1180 (1990).

20. J. Hardy, Adaptive Optics for Astronomical Telescopes, Oxford series in optical and imaging sciences (Oxford University Press, 1998).

21. L. C. Andrews, R. L. Phillips, R. J. Sasiela, and R. R. Parenti, "Strehl ratio and scintillation theory for uplink gaussian-beam waves: beam wander effects," Optical Engineering 45, 076001-076001-12 (2006).

22. H. T. Yura and R. A. Fields, "Level crossing statistics for optical beam wander in a turbulent atmosphere with applications to ground-to-space laser communications," Appl. Opt. 50, 2875-2885 (2011).

23. B. Epple, "Simplified channel model for simulation of free-space optical communications," Journal of Optical Communications and Networking 2, 293-304 (2010).

24. "Prediction method of fade dynamics on earth-space paths," Tech. rep., International Telecommunication Union (ITU), Recommendation ITU-R P.1623-1 (2005)

25. A.-R. Camboulives, M.-T. Velluet, S. Poulenard, L. Saint-Antonin, and V. Michau, "Optical ground station optimization for future optical geostationary satellite feeder uplinks," (2017).

26. J. H. Shapiro and A. L. Puryear, "Reciprocity-enhanced optical communication through atmospheric turbulence. part i: Reciprocity proofs and far-field power transfer optimization," J. Opt. Commun. Netw. 4, 947954 (2012).

27. C. Robert, J.-M. Conan, and P. Wolf, "Impact of turbulence on highprecision ground-satellite frequency transfer with two-way coherent optical links," Phys. Rev. A 93, 033860 (2016). 